\title{
Heavy flavours analysis in proton-proton collisions at the LHC with ALICE
}

\author{
Giacomo Ortona*t \\ INFN Torino \& Università Degli Studi di Torino \\ E-mail: brtonade.infn.it
}

\begin{abstract}
The extremely high energies that will be reached at the Large Hadron Collider (LHC) at CERN will allow studying the production of heavy flavours (beauty and charm) with high statistics in both proton-proton and $\mathrm{Pb}-\mathrm{Pb}$ collisions. The study of open charm (D) and beauty (B) mesons in $\mathrm{Pb}-\mathrm{Pb}$ collisions will be a powerful tool to investigate the production of heavy flavours and their interaction with the medium produced in such collisions (QGP). Heavy flavour yields will provide also a normalization for quarkonia production. We will present a general overview of the ALICE collaboration heavy flavour program, then we will focus on the analysis and reconstruction strategies developed for the study of the charmed (D) mesons by the ALICE collaboration for proton-proton collisions, with special emphasis on the charged D mesons. Finally, some expected results obtained with MonteCarlo production will be shown.
\end{abstract}

XLVIII International Winter Meeting on Nuclear Physics, BORMIO2010

January 25-29, 2010

Bormio, Italy

\footnotetext{
* Speaker.

${ }^{\dagger}$ For the ALICE collaboration.
} 


\section{Introduction}

At the Large Hadron Collider (LHC) at CERN first proton-proton collisions at an energy of $\sqrt{s_{N N}}=900 \mathrm{GeV}$ have been delivered in November 2009 and since March 2010 at an energy of $\sqrt{s_{N N}}=7 \mathrm{TeV}$, while first $\mathrm{Pb}-\mathrm{Pb}$ collisions are foreseen in November 2010. The maximum energy LHC will be able to reach is as high as $\sqrt{s_{N N}}=5.5 \mathrm{TeV}$ for Pb-Pb collisions and $\sqrt{s_{N N}}=14 \mathrm{TeV}$ for proton-proton collisions, opening up a new domain for the study of strongly interacting matter in conditions of high temperature ( 3 to 5 times higher than the critical temperature) and high energy density ( 15 to $60 \mathrm{GeV} / \mathrm{fm}^{3}$ ).

QCD calculations [ $[$ ] at high temperature and high energy density predict in such conditions the formation of a deconfined state of matter, called Quark-Gluon Plasma. In the study of the properties of the produced (deconfined) state, heavy quarks play a crucial role. Heavy quarks and hard partons, abundantly produced at LHC energies in the initial hard scattering processes, are sensitive probes of the medium formed in the collisions as they may lose energy by gluon radiation while propagating through the medium itself.

At LHC energy, charm and beauty will be abundantly produced. The cross-sections at LHC are expected to increase by about a factor of 10 for charm and 100 for beauty with respect to RHIC. The baseline production cross-section of $Q \bar{Q}$ pairs for ALICE simulation studies have been calculated in the framework of collinear factorization and $\mathrm{pQCD}[2]$, including the nuclear modification of the parton distribution functions (PDFs)[[]]. The expected $c \bar{c}$ and $b \bar{b}$ production yields for pp collisions at the maximum energy available at the LHC $\left(\sqrt{s_{N N}}=14 \mathrm{TeV}\right)$ are 0.16 and 0.0072 , respectively[四]. For the $5 \%$ most central $\mathrm{Pb}-\mathrm{Pb}$ collisions at $\sqrt{s_{N N}}=5.5 \mathrm{TeV}$, the expected yields are 115 and 4.6 respectively, but it has to be noted that these predictions have large uncertainties, of about a factor 2 to 3, depending on the choice of the quark masses and QCD scales.

\section{The ALICE experiment at the LHC}

The ALICE experiment, described in detail in[6], performs very well for heavy flavour measurements $[\square]$. Experimentally, the two key elements for a rich heavy-flavour program are: good tracking/vertexing and good particle identification. In ALICE, Particle tracking relies on the six concentric layers of high resolution silicon detectors of the Inner Tracking System (ITS) ${ }^{1}$, a large volume of time projection chamber (TPC) ${ }^{2}$, and a high granularity transition-radiation detector (TRD). The ALICE detection strategy for charm and beauty hadrons relies on resolving secondary detached vertices reconstructed from tracks with large impact parameters $(\mathrm{d} 0)^{3}$. The precision on impact parameter measurement is mainly provided by the two innermost ITS layers (SPD) in the bending plane ( $\mathrm{r} \phi$ ) and the two intermediate ITS layer (SDD) for z-coordinate. A resolution $\sigma_{d 0}$ better than $60 \mu \mathrm{m}$ in the bending plane is achieved for tracks with $p_{T}>1.5 \mathrm{GeV} / \mathrm{c}$. This resolution is enough to distinguish particles coming from the displaced decay vertices of the D mesons ( $c \tau \sim 100 \mu \mathrm{m}$, see table $\mathrm{W})$ and B mesons $(c \tau \sim 500 \mu \mathrm{m})$

\footnotetext{
${ }^{1}$ Two innermost layers equipped with silicon pixel (SPD) plus two middle layers of silicon drift detectors (SDD), and two outermost layers of silicon strip detectors (SSD)

${ }^{2} \mathrm{~A}$ large drift chamber with multiwire proportional chamber (MWPC) endcaps[ [6]

${ }^{3}$ the impact parameter being the distance of closest approach of a particle trajectory to the primary vertex
} 


\begin{tabular}{|c|c|c|c|}
\hline Meson & Decay channel & $c \tau$ & BR \\
\hline$D^{0}$ & $D^{0} \rightarrow K^{-} \pi^{+}$ & $122.9 \mu m$ & $(3.91 \pm 0.05) \%$ \\
\hline$D^{0}$ & $D^{0} \rightarrow K \pi \pi \pi$ & $122.9 \mu m$ & $(8.14 \pm 0.20) \%$ \\
\hline$D^{+}$ & $D^{+} \rightarrow K^{-} \pi^{+} \pi^{+}$ & $311.8 \mu m$ & $(9.29 \pm 0.25) \%$ \\
\hline$D_{s}^{+}$ & $D_{s}^{+} \rightarrow K^{+} K^{-} \pi^{+}$ & $149.9 \mu m$ & $(5.50 \pm 0.28) \%$ \\
\hline$D^{*+}$ & $D^{*+} \rightarrow D^{0} \pi^{+}$ & & $(61.9 \pm 2.9) \%$ \\
\hline
\end{tabular}

Table 1: Golden Channels for the reconstruction of open charm mesons[[]

Particle identification is performed, in the central rapidity region $(-0.9<\eta<0.9)$, over the full azimuth by a dE/dx measurement in the tracking detectors (TPC and ITS), via time of flight measurement using Time of Flight detector (TOF) and transition radiation measurement in the Transition Radiation Detector (TRD, although its installion is not fully complete yet, so part of the TRD azimuthal coverage is missing). Muons are identified in the Forward Muon Spectrometer in the acceptance $-4<\eta<-2.5$

\section{Heavy Flavour measurement}

Open charm mesons will be measured through their hadronic decays in fully reconstructed topologies. The strategy to reduce the large combinatorial background is based on the selection of displaced-vertex topologies, i.e. large separation between the primary and the secondary vertices, an impact parameter value in the order of $\sim 100-500 \mu \mathrm{m}$ and good alignment between the reconstructed D meson momentum and the flight line, being the flight line the line passing through both the primary and the secondary vertex. The angle between reconstructed D meson momentum and the flight line is called pointing angle.

The golden channels that will be studied are listed in table $\square$.

After the reconstruction, an invariant mass analysis is used to extract the raw signal yield, and then corrections for the detector acceptance, for selection efficiency and reconstruction efficiency are applied.

\section{$D^{0}$ reconstruction}

The most promising channel for the study of $D^{0}[\mathbb{Z}, \mathbb{8}]$ is the channel $D^{0} \rightarrow K^{-} \pi^{+}$, although also the 4-prongs decay $D^{0} \rightarrow K \pi \pi \pi$ will be studied. The selection strategy is based on the application of 2 different cuts: the product of the impact parameter of the two tracks $\left(d_{0}^{K} \times d_{0}^{\pi}\right)$ and the cosinus of the pointing angle ${ }^{4}$ of the reconstructed $D^{0}$ meson. Figure $\square$ shows a sketch of this decay topology. The goal of this selection is to increase the significance (the ratio $\frac{S}{\sqrt{S+B}}$, where $\mathrm{S}$ is the signal and B the background). The effect of this cuts on the significance is showed in figure $\square$. So far more then $1.4 \cdot 10^{8}$ events in proton-proton collisions at $7 \mathrm{TeV}$ have been analyzed. From the study of this data we expect a significance of 57 for the decay channel $D^{0} \rightarrow K^{-} \pi^{+}$after $10^{9}$ events, considering only candidates with $p_{T}>2 \mathrm{GeV}$.

\footnotetext{
${ }^{4}$ If the reconstructed particle is a real D meson, then $\cos \theta_{\text {point }} \approx 1$
} 


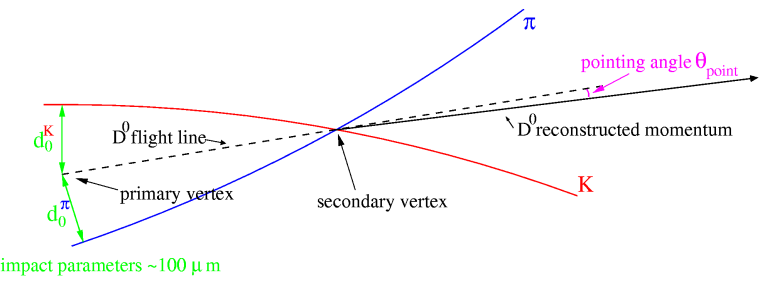

Figure 1: Schematic representation of the decay $D^{0} \rightarrow$ $K^{-} \pi^{+}$

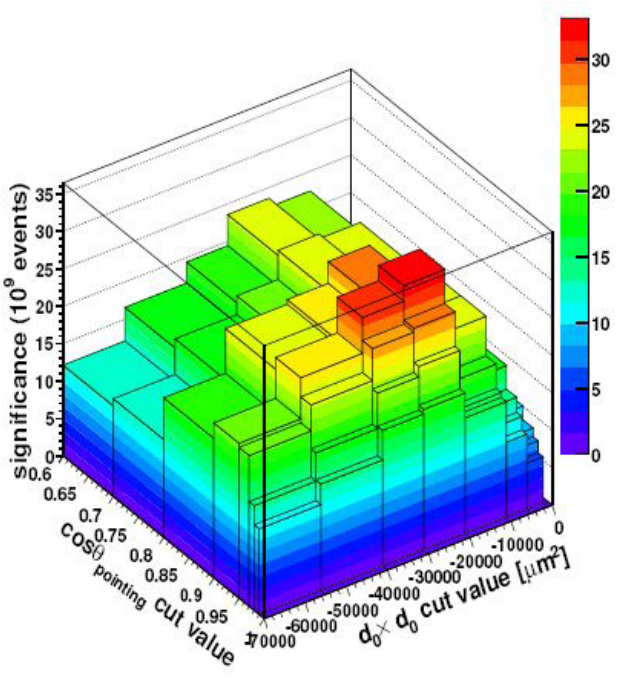

Figure 2: $D^{0}$ significance normalized to $10^{9}$ events ( 1 year of data taking at $14 \mathrm{TeV}$ ), in function of cut variables

\section{$D^{+}$reconstruction}

The $D^{+}$meson will be reconstructed in the channel $D^{+} \rightarrow K^{-} \pi^{+} \pi^{+}$[Q] ]. With respect to the decay $D^{0} \rightarrow K^{-} \pi^{+}$this topology is affected by higher combinatorial background. On the other hand, as showed in table $\mathbb{W}$, the branching ratio of this decay is higher and the $D^{+}$has longer life time $(c \tau \sim 310 \mu \mathrm{m})$. Thus we can use a cut on the distance between primary and secondary vertices to remove combinatorial background. Triplets with the correct charge sign combination will be built, then the main cuts for a selection strategy to separate the signal from the huge combinatorial background for the $D^{+}$are therefore the distance between the primary and secondary vertices and the cosinus of the pointing angle of the reconstructed meson. To perform this selection, an extremely good vertexing resolution, both on the primary and secondary vertex must be reached. $\mathrm{In} \mathrm{Pb}-\mathrm{Pb}$ collisions, the identification of the kaon is also used to reduce combinatorial background. The expected performance on vertex resolution of ALICE is shown in figure B. Figure $\$$ shows the expected statistical error after 1 year of data taking. The expected significance after $10^{9}$ events at 7 $\mathrm{TeV}$ estrapolated from $1.4 \cdot 10^{8}$ analyzed events for candidates with $p_{T}>2 \mathrm{GeV}$ is expected to be 49 for this decay channel.

\subsection{Open Beauty measurement}

Open beauty mesons will be studied through their inclusive semi-leptonic channels $B \rightarrow e+X$ and $B \rightarrow \mu+X[\square]$. The semielectronic channel will be studied by the detectors in the central rapidity region (TPC, ITS, TRD). The selection will be based on 3 selection steps: at first, electron PID will reject most of the hadrons (expecially pions), then a cut on the impact parameter to reduce background electrons and misidentified charged pions will be applied and finally will be performed a cut on transverse momentum to reject electrons from charm decay.

The semimuonic channels will be studied in the ALICE muon specrometer. Muons will be divided in 2 different samples: the first containing muons of opposite sign coming from the same b quark 


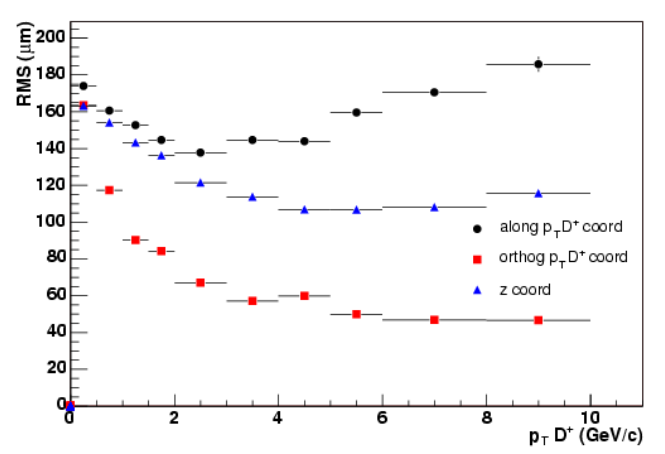

Figure 3: Resolution on the $D^{+}$decay vertex. Black dots: longitudinal direction with respect to the $D^{+}$ flight direction. Red squares: Orthogonal to $D^{+}$ flight direction. Blue triangles: along $\mathrm{z}$ coordinate

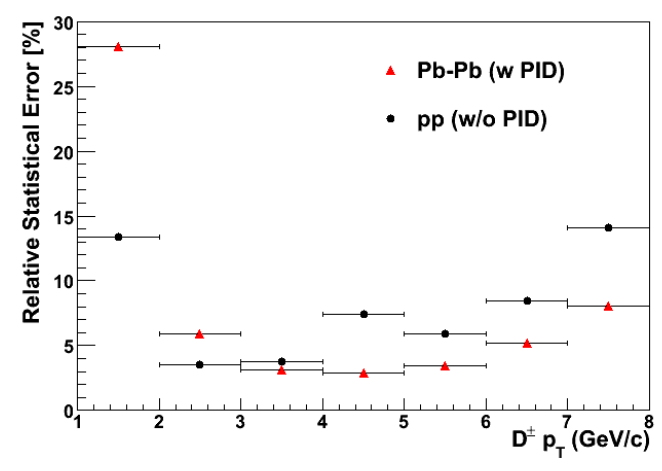

Figure 4: Relative statistical error for $D^{+}$after 1 year of data taking in pp (14 TeV) collisions without any $\mathrm{PID}$ and $\mathrm{Pb}-\mathrm{Pb}$ (5.5 ATev) collisions assuming perfect PID.

( $\left.B D_{\text {same }}\right)$ which dominates the low mass region $\left(M_{\mu^{+} \mu^{-}}<5 \mathrm{GeV}\right)$ and the second with muon pairs of the same sign containing muons originating each from a different $b$ quark in the couple $b \bar{b}$, dominating the region $5<M_{\mu^{+} \mu^{-}}<20 \mathrm{GeV}$. The main background sources, decays from $\pi^{ \pm}, K^{ \pm}$ and charm, will be subtracted through fit techniques. After 1 year of data taking in p-p collisions ( $10^{9}$ events) the relative statistical error, for electron from $\mathrm{B}$ decay reconstructed in the central barrel, is expected to be smaller than $10 \%$ for electron $p_{T}$ up to $12 \mathrm{GeV} / \mathrm{c}$ [प]].

\section{Energy Loss}

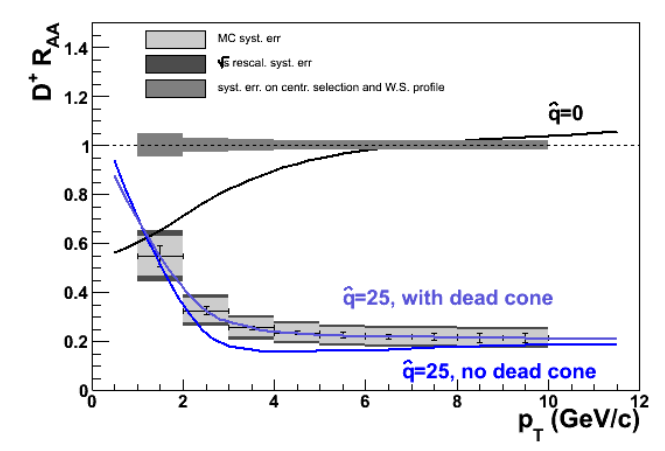

Figure 5: Nuclear modification factor for $D^{+}$meson, 1 year of data taking, energy loss calculation from[ए]]

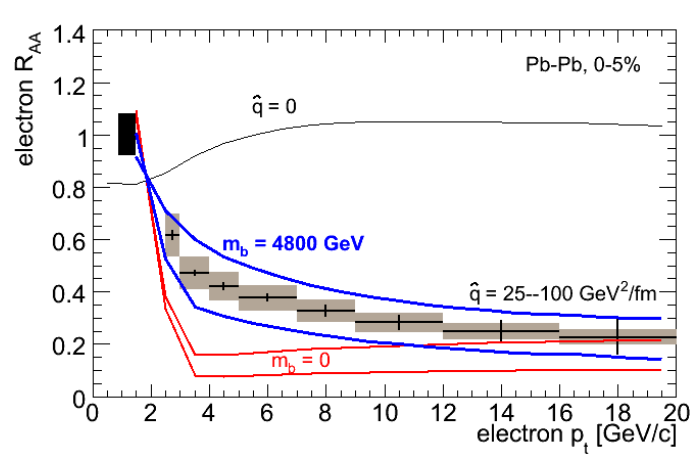

Figure 6: Nuclear modification factor for electrons from $B$ meson decays. 1 year of data taking, energy loss calculation from[ए]]

Parton energy loss is expected to depend strongly on the properties of the medium, parametrized by $\hat{q}[\mathbb{W}]$ (a measurement of the medium density in the BDMPS approach), and of the probe (colour charge, mass) travelling through it. Charm and beauty quarks are expected to be qualitatively different probes with respect to light partons, as their energy loss is reduced due to mass effects (dead cone effect[[2]). In figure [ $]$ is shown the expected measure of the nuclear modification factor after 1 year of data taking for the $D^{+}$and the electrons coming from $B$ decay (figure 6 ). The ratio 


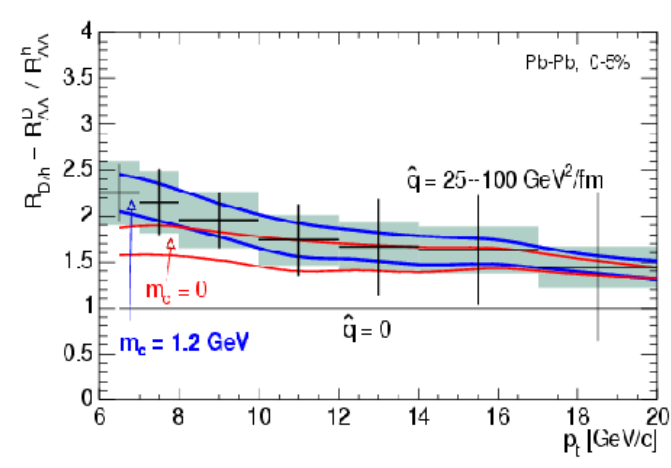

Figure 7: Heavy-to-light ratio $R_{D h}(P t)=\frac{R_{A A}^{D}(P t)}{R_{A A}^{h}(P t)}$, 1 year of data taking, energy loss calculation from[1]]

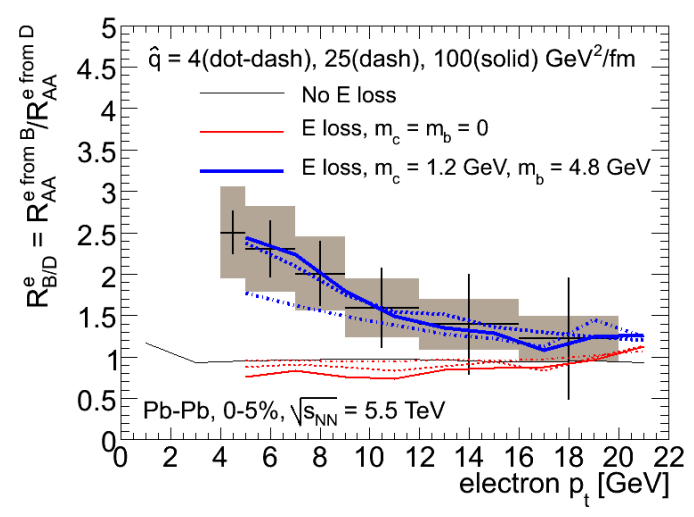

Figure 8: $R_{B D}=\frac{R_{A A}^{B}(P t)}{R_{A A}^{D}(P t)}, 1$ year of data taking, energy loss calculation from[ए]]

$R_{D h}(P t)=\frac{R_{A A}^{D}(P t)}{R_{A A}^{h}(P t)}$, showed in figure $\square$ is an interesting probes of the colour charge dependance of the energy loss, as light hadrons mainly come from gluons, carrying a stronger colour charge. Also, the ratio $R_{B D}(P t)=\frac{R_{A A}^{B}(P t)}{R_{A A}^{D}(P t)}$ (figure $\mathbb{E}$ ) is a probe of of the mass dependance of the energy loss, as $B$ and $D$ come from objects with the same colour charge but different mass. As shown in figures $1,6, \square, \mathbb{Q}$, after 1 year of data taking at nominal luminosity, ALICE is expected to be able to discriminate between different models and dependances (small or large $\hat{q}$ values, quark masses in medium ranging from $m_{c} \approx m_{b} \approx 0$ to $m_{c} \approx 1.2 \mathrm{GeV}$ and $m_{b} \approx 4.8 \mathrm{GeV}$ )

\section{5. conclusions}

With its excellent tracking, vertexing and particle identification capabilities, ALICE has very promising prospectives for the studies of heavy flavour production. MonteCarlo silumations at 14 $\mathrm{TeV}$ show that in 1 year of p-p collisions at nominal luminosity it is feasible to reconstruct $\mathrm{D}$ and B mesons with good significance $\left(\sim 30\right.$ for $\left.D^{0}\right)$ and small relative statistical error (less than $10 \%$ for $D^{+}$with $2 \mathrm{GeV} / \mathrm{c}<p_{T}<7 \mathrm{GeV} / \mathrm{c}$ and for B with electrons $p_{T}<12 \mathrm{GeV} / \mathrm{c}$ down to $p_{T} \sim 1 \mathrm{GeV} / \mathrm{c}$ ). The first results obtained from collisions at $7 \mathrm{TeV}$ are also confirming the good capabilities of ALICE in the measurement of the heavy flavoured particles.

\section{References}

[1] F. Karsch, Lattice QCD at High Temperature and Density [hep-lat/0106019] and M. Cheng et al., QCD equation of state with almost physical quark masses Phys. Rev. D77,014511 (2008).

[2] M.L. Mangano, P. Nason and G. Radolfi, Heavy-quark correlations in hadron collisions at next-to-leading order, Nucl. Phys. B373 295 (1992).

[3] K.J Eskola, V.J Kolhinen, C.A. Salgado, The scale dependent nuclear effects in parton distributions for practical applications, Eur. Phys. J. C9, 61(1999).

[4] N. Armesto et al., Heavy-ion collisions at the LHC - Last call for predictions, J. Phys. G35 054001, [arXiv:0711.0974] (2008).

[5] C. Amsler et al. (Particle Data Group), PL B667. 1 (2008) and 2009 partial update for 2010 edition 
[6] ALICE collaboration, The Alice Experiment at CERN LHC, JINST0803: S08002 (2008).

[7] ALICE Collaboration, Physics Performance Report vol. II, CERN/LHCC 2005-030 and J. Phys. G32 1295 (2006).

[8] A. Dainese, Ph. D. Thesis [nucl-ex/0311004].

[9] E. Bruna, Ph. D. Thesis [nucl-ex/0703005v2].

[10] A. Dainese, Charm and beauty at the LHC, Nucl. Phys. A738 [nucl-ex/ 0609042 v2] (2007).

[11] N. Armesto et al., Testing the color charge and mass dependence of parton energy loss with heavy-to-light ratios at BNL RHIC and CERN LHC, Phys. Rev. D71 054027 (2005).

[12] D. Kharzeev and Yu. Dokshitzer, Heavy-quark colorimetry of QCD matter, Phys. Lett. B519 (2001). 